\title{
Integrity-Directed Sensor Fusion by Robust Risk Assessment of Fault-Tolerant Interval Functions
}

\author{
Olivier Bilenne \\ Multitel ASBL \\ Mons, Belgium \\ bilenne@multitel.be
}

\begin{abstract}
This study is concerned with the problem of static parameter estimation by robust sensor fusion in the context of applications requiring, for safety reasons, a high guarantee on the reliability of the results. The integrity certification process consists of providing confidence intervals for the unknown parameter with extremely low risks of error. The concept of integrity certification is formalized for robust sensor fusion methods based on interval arithmetics, including the widely-used fault-tolerant interval functions introduced by $K$. Marzullo and U. Schmid. Random Boolean variables with given bounds on their a priori probabilities are first considered in the Boolean logic setting. Worst-case reasoning leads to the robust derivation of integrity bounds on logical combinations of these variables. Next, integrity bounds on the Marzullo and Schmid functions are derived from the properties of a non-connex Boolean extension of Marzullo's function.
\end{abstract}

Keywords: Robust estimation, integrity, fault-tolerant interval functions, probabilistic inference, Boole-Fréchet bounds.

\section{Introduction}

The present work was motivated by the increasing demand for automatisation of the traffic monitoring of transport systems. The control of many navigation systems relies on the estimation of parameters, and the safety of the parameter estimation procedures is a vital issue. The estimation algorithms must provide safe confidence intervals in which the mobile lies with an extremely low risk of error. The risk of providing misleading information is measured by a quality measure related to the safe use of the system, and called integrity. The integrity of a system measures the probability with which misleading information is issued. The International Norm Standards (e.g. the International Electrotechnical Commission IEC61508and the CENELEC EN50126standards) define Safety Integrity Levels (SIL), expressing the maximum tolerated frenquency of misleading information. In a safety context, these error rates must generally be kept very small, namely $10^{-7}$ (SIL3) or even $10^{-9}$ (SIL4) error per hour.

This study aims at formalizing the important concept of integrity certification given by the International
Norm Standards for robust interval-based sensor fusion methods, such as the fault-tolerant interval functions. Although fault-tolerant interval functions have been extensively used in mobile positioning or clock synchronization problems [1][2], their integrity has yet to be proven when the majority of sensors can not be guaranteed to provide $100 \%$-sure confidence intervals. In this paper, a safe confidence degree is allotted to each confidence interval. This modelling of the probability distributions, combining intervals and confidence degrees and mainly inspired from [3], was adopted in other studies such as [4].

Supposing that the frequency at which the information is ditributed is known, the process of integrity certification of a system can be done by evaluating and limiting the probability of error of each successive piece of information $x$. Using the traditional a posteriori approach of estimation theory [5], the risk assessment problem could be solved by guaranteeing that the posterior probabilities $P(x=t r u e \mid y)$ are larger than some lower bound $I$ for all observations $y$. Indeed, the expected reliability of $x$ would by bounded by $I$ :

$$
E_{y}[P(x=\text { true } \mid y)] \geq I
$$

Unfortunately, this approach generally leads to robustness issues as it requires a good knowledge of the joint probability distributions of $x$ and $y$, which is seldom the case in real applications. For instance, the faulttolerant interval estimation method presented in [4], and based on a sensor fusion method inspired from the Evidence Theory, offers no robust guarantee in terms of mean error rates. In this study, we prefer to adopt a robust 'worst-case' approach. We consider various possible working modes $\phi$ for the sensors, and make sure that the probability $P(x=$ true $\mid \phi)$ is greater than $I$ for all possible scenarios $\phi$, or equivalently, for the mode minimizing this probability, independently from any observation. With marginalization over the modes $\phi$, this a priori worst-case approach ensures the lower bound $I$ on the expected reliability of $x$ :

$$
E_{\phi}[P(x=\text { true } \mid \phi)] \geq \min _{\phi} P(x=\text { true } \mid \phi) \geq I .
$$

It should be noted that the worst-case approach involves that the performances of the method presented in this study are a priori features which do not rely on future observations and can be predicted offline. Sec. 4 
shows how these features allow to derive integrity specifications for flexible control process programs robust to individual sensor failures, such as the well-known fault-tolerant functions proposed by K. Marzullo in [1] and U. Schmid in [6].

Secs. 2-3 first focus on the more general problem of certifying, using a robust worst-case approach in a strictly Boolean setting, the integrity of logical combinations of random Boolean variables with uncertain stochastic properties, when safe bounds on the a priori probabilities of the variables are assumed to be known.

\section{$2 \quad$ Integrity of Boolean variables}

\subsection{Boolean variables and working modes}

We consider the problem of estimation of a system subject to the observations of various sensors. The reliability of sensors, and more generally the validity of the models used for estimating the system, are represented by a set of ordered Boolean variables $x_{1}, x_{2}, \ldots, x_{n}$. We suppose that the system may operate under various working conditions and failure scenarios. As a consequence, the stochastic properties of the variables can only be known with degrees of uncertainty. These modelling uncertainties are symbolized by a single parameter $\phi$, called the mode, which models the joint uncertainties of the probability distributions of all the variables. Let $\Phi$ be the set of all the possible modes. We denote by $P_{\phi}(x)$ the conditional probability ${ }^{1}$ of a variable $x$ to take the value true under a given mode $\phi$. The conditional probabilities of a same variable may vary strongly depending on the operating mode of the system. Therefore, it is assumed that a pre-treatment is done on the data and observations to detect the obvious outliers and sensor failures, so that the mode of the system is guaranteed to belong to a subset $\Phi_{0}$ of $\Phi$, on which reliability constraints and limiting bounds on the stochastic properties of the variables are known. Adopting a robust approach, we decide to include in $\Phi_{0}$ all the possible modes presenting the variables with stochastic properties adequate to the reliability constraints of the problem. Sec. 2.2 shows how these constraints can be formulated.

\section{$2.2 \quad$ Integrity}

The integrity of a Boolean variable $x$ is defined as the minimum probability over $\Phi_{0}$ that $x$ is true:

$$
\operatorname{Integrity}(x)=\min _{\phi \in \Phi_{0}} P_{\phi}(x)
$$

Equivalently, the worst-case risk of a variable can be defined as the maximum probability over $\Phi_{0}$ that the variable is false. The recent developments of S. Ferson's probability boxes [7] and A. Neumaier's fuzzy clouds [8] are a sign of the growing interest for the

\footnotetext{
${ }^{1}$ Note that, in order to simplify the presentation, all conditional probabilities are written under the compact syntax $P_{\phi}(x)$ rather than the more popular equivalent notation $P(x \mid \phi)$.
}

representation and exploitation of variables with uncertain stochastic properties. In this study, the probability of a variable under each possible mode $\phi$ of $\Phi_{0}$ lies in a probability interval. Since integrity certification problems are concerned with lower probability bounds, it is assumed that the upper probability bound of a variable $x$ is generally set to 1 and that the probability interval of $x$ is of the form

$$
P_{\phi}(x) \in\left[\min _{\bar{\phi} \in \Phi_{0}} P_{\bar{\phi}}(x), 1\right]
$$

for all $\phi \in \Phi_{0}$. The objective of the next section is to find safe probability intervals for Boolean formulas. Boolean formulas are constructed by combining propositional variables, logical connectives $(\neg, \wedge, \vee)$, and parentheses. From there, we will see in Sec. 4 how the results obtained in Sec. 2.3 in the propositional logic framework apply to interval arithmetics by allowing to derive integrity bounds on fault-tolerant interval functions.

\subsection{Combining Boolean terms}

\subsubsection{The Boole-Fréchet bounds for disjunc- tion and conjunction}

The most simple Boolean formulas are the conjunction $(\wedge)$ and the disjunction $(\vee)$ operators. The probability bounds of the conjunction and the disjunction of two variables with known probability intervals are given by the Boole-Fréchet bounds. These bounds were found by G. Boole in the nineteenth century, and later brought up to date by M. Fréchet [9]. Let $x_{1}$ and $x_{2}$ be two Boolean variables with respective probability bounds $P_{\phi}\left(x_{1}\right) \in\left[a_{1}, b_{1}\right]$ and $P_{\phi}\left(x_{2}\right) \in\left[a_{2}, b_{2}\right]$, with $0 \leq a_{1} \leq b_{1} \leq 1$ and $0 \leq a_{2} \leq b_{2} \leq 1$, we have

$$
\begin{aligned}
& P_{\phi}\left(x_{1} \vee x_{2}\right) \in\left[\max \left(a_{1}, a_{2}\right), \min \left(b_{1}+b_{2}, 1\right)\right], \\
& P_{\phi}\left(x_{1} \wedge x_{2}\right) \in\left[\max \left(0, a_{1}+a_{2}-1\right), \min \left(b_{1}, b_{2}\right)\right] .
\end{aligned}
$$

As the study is mainly concerned with integrity problems, we rewrite the Boole-Fréchet bounds for probability ranges of the type (4) by setting $b_{1}=b_{2}=1$. The worst-case bounds for the probabilities of the disjunction and conjunction of two Boolean variables $x_{1}$ and $x_{2}$ are then given by

$$
\begin{aligned}
& P_{\phi}\left(x_{1} \vee x_{2}\right) \in\left[\max \left(\min _{\phi \in \Phi_{0}} P\left(x_{1}\right), \min _{\phi \in \Phi_{0}} P\left(x_{2}\right)\right), 1\right], \\
& P_{\phi}\left(x_{1} \wedge x_{2}\right) \in\left[\max \left(0, \min _{\phi \in \Phi_{0}} P\left(x_{1}\right)+\min _{\phi \in \Phi_{0}} P\left(x_{1}\right)-1\right), 1\right] .
\end{aligned}
$$

The conjunction operator helps to better identify the stochastic parameters that rule the system inside their definition spaces. A special case is when one variable is (locally) a more 'accurate' observer of those parameters than the other, in the sense that the realization of this variable implies the realization of the other. Then the conjunction has the property to select the most informative variable amongst them. An illustration of this property in the context of interval arithmetic is given in Sec. 4, where the automatic selection of 
the most 'accurate' variable is the basis of the faulttolerant functions of Marzullo and Schmid.

The disjunction operator allows to reach high integrity levels by exploiting additional knowledge on the correlations between the variables. This property is discussed in the next section.

\subsubsection{Exploitation of dependence constraints}

Various ways of representing the dependence between two Boolean variables are listed in [10]. The modelling proposed in this section is adapted to the case when the probabilities of the variables are uncertain. Since the probability of the conjunction and the disjunction of two variables $x_{1}$ and $x_{2}$ are bound by the equation

$$
P_{\phi}\left(x_{1} \wedge x_{2}\right)+P_{\phi}\left(x_{1} \vee x_{2}\right)=P_{\phi}\left(x_{1}\right)+P_{\phi}\left(x_{2}\right),
$$

the dependence between two Boolean variables can be completely characterized by a scalar measure [10]. We denote the worst-case risk of variable $x_{k}$ by

$$
\alpha_{k}=\max _{\phi \in \Phi_{0}} P_{\phi}\left(\neg x_{k}\right),
$$

for $k=1,2$. The correlations can be modelled for instance by a function $\alpha$ which takes its values in $\left[0, \min \left(\alpha_{1}, \alpha_{2}\right)\right]$ and gives, for each mode $\phi$, the risk $\alpha(\phi)=P_{\phi}\left(\neg\left(x_{1} \vee x_{2}\right)\right)$ associated with the disjunction of $x_{1}$ and $x_{2}$. We have

$$
\begin{aligned}
& P_{\phi}\left(x_{1} \vee x_{2}\right)=1-\alpha(\phi), \\
& P_{\phi}\left(x_{1} \wedge x_{2}\right)=1-\left(P_{\phi}\left(\neg x_{1}\right)+P_{\phi}\left(\neg x_{2}\right)-\alpha(\phi)\right)
\end{aligned}
$$

Given a mode $\phi, x_{1}$ and $x_{2}$ are perfectly correlated if $\alpha(\phi)=\min \left(P_{\phi}\left(\neg x_{1}\right), P_{\phi}\left(\neg x_{2}\right)\right)$, positively correlated if $P_{\phi}\left(\neg x_{1}\right) P_{\phi}\left(\neg x_{2}\right)<\alpha(\phi)<\min \left(P_{\phi}\left(\neg x_{1}\right), P_{\phi}\left(\neg x_{2}\right)\right)$, non-correlated if $\alpha(\phi)=P_{\phi}\left(\neg x_{1}\right) P_{\phi}\left(\neg x_{2}\right)$, and negatively correlated if $0 \leq \alpha(\phi) \leq P_{\phi}\left(\neg x_{1}\right) P_{\phi}\left(\neg x_{2}\right)$. Extending the idea of making assumptions on the nature of the dependencies (maximum, minimum, nonnegative correlations or independence) [11] to problems where only lower bounds on the probabilities of the variables are known, we propose to use the information on the correlations of $x_{1}$ and $x_{2}$ to restrict the range of $\alpha(\phi)$ to a sub-interval $\left[\alpha^{c}, \alpha^{d}\right]$ of $\left[0, \min \left(\alpha_{1}, \alpha_{2}\right)\right]$, and derive tighter lower bounds on the probabilities of the disjunction and conjunction :

$$
\begin{aligned}
& P_{\phi}\left(x_{1} \vee x_{2}\right) \in\left[1-\alpha^{d}, 1\right] \\
& P_{\phi}\left(x_{1} \wedge x_{2}\right) \in\left[\max \left(0,1-\left(\alpha_{1}+\alpha_{2}-\alpha^{c}\right)\right), 1\right],
\end{aligned}
$$

with $0 \leq \alpha^{c} \leq \alpha^{d} \leq \min \left(\alpha_{1}, \alpha_{2}\right)$. Under the idealistic hypothesis of independent variables, the upper bounds on the risks of the disjunction given by Eq. (7) vanish from $\min \left(\alpha_{1}, \alpha_{2}\right)$ to $\alpha_{1} \alpha_{2}$, which are respectively of first and second order in terms of $\alpha_{k}$. Consequently, when dealing with high integrities, that is for small values of the risks $\alpha_{1}$ and $\alpha_{2}$, disjunction can lower significantly the worst-case risk of nearly-independent Boolean variables, with respect to the individual risks of these variables. On the other hand, it can be seen from Eq. (14) that the interest of bound $\alpha^{c}$ is less obvious, as conjunction leads at best to the reduction of the worst-case risk by half. Therefore, in order to simplify, this works will only take Eq. (13) into consideration and count on the existence of a bound $\alpha^{d}$, which will ideally lie close to the independence value $\alpha_{1} \alpha_{2}$, while $\alpha^{c}$ will be set to 0 by default, and now-redundant Eq. (14) will be intentionally ignored. This modelling approach of the correlations can easily be extended to more than two variables by providing lower bounds on the probabilities of $n$-ary disjunctions of the variables.

\subsubsection{Integrity bounds on more complex com- binations}

This section briefly discusses the derivation of probability bounds on formulas more complex than simple conjunctions and disjunctions. We are in fact confronted with a probabilistic inference problem, where one must determine the logical consequences, on the probability of a given formula, of the probability constraints for the Boolean variables (and possibly for disjunctions of these variables). Safe probability bounds on a formula can be computed by repeatedly and systematically applying the Boole-Fréchet bounds $(5,6)$ to pairs of terms. However, these bounds will generally not be the best possible bounds if the formula presents repetitions of the same variables, as in $\left(x_{1} \wedge x_{2}\right) \vee\left(x_{2} \wedge x_{3}\right) \vee\left(x_{3} \wedge x_{1}\right)$ for instance, where all the variables are repeated twice. The loss of optimality would be due to the multiple introduction in the calculation of the uncertanties of the repeated variables [12][13]. [10] lists various alternative strategies developed to handle formulas with repeated paramaters. These strategies include subinterval reconstitution (partition of the probability intervals in subintervals [14]), range sampling (variant of Monte Carlo simulations), and the rule-based method, using local inference rules and illustrated in Sec. 3.3. The inference problem was formalized in [15], and can be solved by linear programming for fixed values of the probability bounds of the integrity constraints. However, the derivation of a systematic and efficient generic solution for parametric values of the integrity bounds is an open problem which goes beyond the scope of this paper. It should be noted that solutions have been proposed in the literature, e.g. in [16].

The next section proposes a predicate formula $G_{n}^{f}$ as an answer to the trade-off between the properties of conjunction and disjunction. Safe integrity bounds on this formula are derived from simple inference rules.

\section{The predicate function $G_{n}^{f}$}

\subsection{Definition}

It is assumed that the Boolean variables to which the function is applied can be ordered arbitrarily and denoted $x_{1}, x_{2}, \ldots, x_{k}, \ldots$. They become the terms of a sequence of variables $\left(x_{k}\right)$ and define a corresponding sequence of sets of variables $\left(X^{k}\right)$, with $X^{k}=\left\{x_{1}, \ldots, x_{k}\right\}$ $(k \geq 1)$. Let

$$
\mathcal{C}_{n}^{f}=\left\{\left\{k_{1}, \ldots, k_{f}\right\}: 1 \leq k_{j} \leq n, j=1, \ldots, f\right\}
$$


be the set of all the possible combinations of $f$ elements of $X^{n}$. We call $G_{n}^{f}\left(x_{1}, \ldots, x_{n}\right)$, or simply $G_{n}^{f}\left(X^{k}\right)$, the $n$-argument predicate defined by the disjunction of the conjunctions of the elements of all the $(n-f)$-combinations of its arguments :

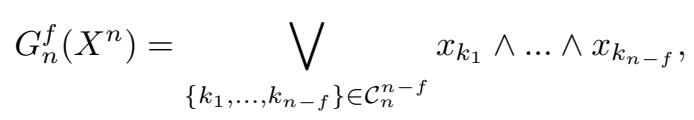

with $0 \leq f \leq n-1$. Practically, $G_{n}^{f}$ takes the value true iif at least $n-f$ of its arguments are true. In other words, $G_{n}^{f}$ tolerates a maximum of $f$ false-valued arguments. The latter property is also met in the context of interval estimation by a well-known interval function proposed by Marzullo, and presented in Sec. 4.2. It should be noted that the formulation of Eq. (16) particularly stresses the similarities with Marzullo's function. The next section presents some other properties of the predicate $G_{n}^{f}$, which will allow to derive integrity bounds for $G_{n}^{f}\left(X^{n}\right)$.

\section{$3.2 \quad$ Properties}

A direct consquence of definition (16) is that the predicate $G_{n}^{f}\left(X^{n}\right)$ always gets a larger chance to be true with increased values of $f$. Predicate $G_{n}^{f+1}\left(X^{n}\right)$ is in fact a consequent of $G_{n}^{f}\left(X^{n}\right)$ and we write

$$
G_{n}^{f}\left(X^{n}\right) \rightarrow G_{n}^{f+1}\left(X^{n}\right) .
$$

We have, if $1 \leq f \leq f^{\prime} \leq n-1$,

$$
\begin{aligned}
& G_{n}^{f}\left(X^{n}\right) \vee G_{n}^{f^{\prime}}\left(X^{n}\right)=G_{n}^{f^{\prime}}\left(X^{n}\right), \\
& G_{n}^{f}\left(X^{n}\right) \wedge G_{n}^{f^{\prime}}\left(X^{n}\right)=G_{n}^{f}\left(X^{n}\right) .
\end{aligned}
$$

The structure of Eq. (16) allows the derivation of a recursive definition for the $G_{n}^{f}$ function. We find, for $0 \leq f \leq n-2$,

$$
\begin{aligned}
G_{1}^{0}\left(X^{1}\right) & =x_{1} \\
G_{n+1}^{0}\left(X^{n+1}\right) & =x_{1} \wedge \ldots \wedge x_{n+1} \\
G_{n+1}^{f+1}\left(X^{n+1}\right) & =G_{n}^{f}\left(X^{n}\right) \vee\left(G_{n}^{f+1}\left(X^{n}\right) \wedge x_{n+1}\right) \\
G_{n+1}^{n}\left(X^{n+1}\right) & =x_{1} \vee \ldots \vee x_{n+1}
\end{aligned}
$$

Eq. (22) is obtained by separating in $G_{n+1}^{f+1}\left(X^{n+1}\right)$ the terms that contain variable $x_{n+1}$ from those which do not involve $x_{n+1}$. We introduce now a second predicate $\tilde{G}_{n}^{f}$ by considering the 'dual' formula of Eq. (16). We define $\tilde{G}_{n}^{f}\left(X^{n}\right)$ as the $n$-argument predicate equal to the conjunction of the disjunctions of the elements of all the $(f+1)$-combinations of its arguments :

$$
\tilde{G}_{n}^{f}\left(X^{n}\right)=\bigwedge_{\left\{k_{1}, \ldots, k_{f+1}\right\} \in \mathcal{C}_{n}^{f+1}} x_{k_{1}} \vee \ldots \vee x_{k_{f+1}},
$$

with $0 \leq f \leq n-1$. It is interesting to note from Eqs. $(16,24)$ that the conjunction $\tilde{G}_{n}^{f}\left(X^{n}\right)$ presents $\left(\begin{array}{c}n \\ f+1\end{array}\right)$ terms of arity $f+1$, while the $G_{n}^{f}\left(X^{n}\right)$ function is a disjunction of $\left(\begin{array}{c}n \\ n-f\end{array}\right)$ terms of arity $n-f$. Definition (24) however leads to identical properties for $\tilde{G}_{n}^{f}\left(X^{n}\right)$. We have, if $1 \leq f \leq f^{\prime} \leq n-1$,

$$
\begin{aligned}
& \tilde{G}_{n}^{f}\left(X^{n}\right) \vee \tilde{G}_{n}^{f^{\prime}}\left(X^{n}\right)=\tilde{G}_{n}^{f^{\prime}}\left(X^{n}\right), \\
& \tilde{G}_{n}^{f}\left(X^{n}\right) \wedge \tilde{G}_{n}^{f^{\prime}}\left(X^{n}\right)=\tilde{G}_{n}^{f}\left(X^{n}\right) .
\end{aligned}
$$

Similarly, a recursive definition of $\tilde{G}_{n}^{f}$ can be found :

$$
\begin{aligned}
\tilde{G}_{1}^{0}\left(X^{1}\right) & =x_{1}, \\
\tilde{G}_{n+1}^{0}\left(X^{n+1}\right) & =x_{1} \wedge \ldots \wedge x_{n+1}, \\
\tilde{G}_{n+1}^{f+1}\left(X^{n+1}\right) & =\tilde{G}_{n}^{f+1}\left(X^{n}\right) \wedge\left(\tilde{G}_{n}^{f}\left(X^{n}\right) \vee x_{n+1}\right) \\
& =\tilde{G}_{n}^{f}\left(X^{n}\right) \vee\left(\tilde{G}_{n}^{f+1}\left(X^{n}\right) \wedge x_{n+1}\right), \\
\tilde{G}_{n+1}^{n}\left(X^{n+1}\right) & =x_{1} \vee \ldots \vee x_{n+1},
\end{aligned}
$$

for $0 \leq f \leq n-2$. Again, Eq. (29) is obtained through the isolation in $\tilde{G}_{n+1}^{f+1}\left(X^{n+1}\right)$ of the terms containing $x_{n+1}$, while Eq. (30) is a consequence of Eq. (26) and the distributivity of conjunction over disjunction. In summary, we have

$$
G_{n}^{f}\left(X^{n}\right)=\tilde{G}_{n}^{f}\left(X^{n}\right),
$$

for $n \geq 1$ and $0 \leq f \leq n-1$. In other words, function $G_{n}^{f}$ and $\tilde{G}_{n}^{f}$ are equivalent, and Eq. (24) can be seen as an alternate definition for the predicate $G_{n}^{f}\left(X^{n}\right)$.

Proof. We proceed recursively. From Eqs. (20,27), we find that Eq. (32) is true for $n=1$. Suppose now that $G_{k}^{f}\left(X^{k}\right)=\tilde{G}_{k}^{f}\left(X^{k}\right)$ for some $k \geq 1$ and $0 \leq f \leq k-1$. We see from Eqs. $(21,28)$ and from Eqs. $(23,31)$, that the equivalence is verified for $n=k+1, f=0$ and $n=k+1, f=k$ respectively. Then Eqs. $(22,30)$ and the assumption ensure that Eq. (32) is also true for $n=k+1$ and $0 \leq f \leq n-2$, which completes the proof.

The equivalence between $G_{n}^{f}$ and $\tilde{G}_{n}^{f}$ involves interesting properties for the predicate. Indeed, $G_{n}^{0}$ and $G_{n}^{n-1}$ correspond to the $n$-ary conjunction and disjunction operators respectively $(n \geq 1)$. Dealing with $n-f$-ary conjunctions in Eq. (16), and at the same time with $f+1$-ary disjunctions in Eq. (24), intermediate values of $f(0<f<n-1)$ offer an answer to the trade-off between the accuracy possibilities of the conjunction and the integrity objectives of the disjunction. Moreover, it is important to note that the predicate $G_{n}^{f}$ is fully symmetric with respect to its arguments, and is not redefined according to the observations. The latter property is a necessary condition for a priori integrity certification, as explained in Sec. 1.

\subsection{Integrity bounds}

Eq. (24) allows to derive integrity bounds for the predicate $G_{n}^{f}$. From the equation

$$
\neg G_{n}^{f}\left(X^{n}\right)=\underset{\left\{k_{1}, \ldots, k_{f+1}\right\} \in \mathcal{C}_{n}^{f+1}}{\bigvee} \neg\left(x_{k_{1}} \vee \ldots \vee x_{k_{f+1}}\right),
$$

we subsequently derive an upper bound on the risk

$$
P\left(\neg G_{n}^{f}\left(X^{n}\right)\right) \leq \sum_{\mathcal{C}_{n}^{f+1}}\left[1-\min _{\phi \in \Phi_{0}} P_{\phi}\left(x_{k_{1}} \vee \ldots \vee x_{k_{f+1}}\right)\right]
$$

and and lower bound on the probability of $G_{n}^{f}$

$$
P\left(G_{n}^{f}\left(X^{n}\right)\right) \geq 1-\sum_{\mathcal{C}_{n}^{f+1}} \max _{\phi \in \Phi_{0}} P_{\phi}\left(\neg\left(x_{k_{1}} \vee \ldots \vee x_{k_{f+1}}\right)\right)
$$


In the particular case of idependent variables, Eq. (35) reduces to

$$
P\left(G_{n}^{f}\left(X^{n}\right)\right) \geq 1-\sum_{\left\{k_{1}, \ldots, k_{f+1}\right\} \in \mathcal{C}_{n}^{f+1}} \alpha_{k_{1}} \ldots \alpha_{k_{f+1}},
$$

where factors $\alpha_{k}$ are the worst-case risks of the variables taken independently :

$$
\alpha_{k}=\max _{\phi \in \Phi_{0}} P_{\phi}\left(\neg x_{k}\right)=1-\min _{\phi \in \Phi_{0}} P_{\phi}\left(x_{k}\right) .
$$

One sees from Eq. (36) that the maximum risk associated with $G_{n}^{f}\left(X^{n}\right)$ is of order $f+1$ in $\alpha_{k}$ 's. This risk vanishes with increasing $f$, in agreement with the predictions of Eq. (17), and the target integrity level can be adjusted by selecting various values for $f$.

Eq. (35) can still provide useful bounds on the integrity of $G_{n}^{f}$ when the Boolean variables are not perfectly independent. Consider for example the case of three correlated variables $x_{1}, x_{2}, x_{3}$. It is assumed that constrainsts on the correlations of each pair of variables are known and expressed by equations of the type (13) :

$$
P_{\phi}\left(x_{p} \vee x_{q}\right) \in\left[1-\alpha_{p, q}^{d}, 1\right],
$$

where $(p, q)=(1,2),(2,3),(3,1)$ and $\alpha_{1,2}^{d}, \alpha_{2,3}^{d}, \alpha_{3,1}^{d}$ are the maximum risks allotted to the disjunctions of each pair of variables. The integrity bound on predicate $G_{3}^{1}$ is given by

$$
P_{\phi}\left(G_{3}^{1}\left(x_{1}, x_{2}, x_{3}\right)\right) \geq 1-\left(\alpha_{1,2}^{d}+\alpha_{2,3}^{d}+\alpha_{3,1}^{d}\right) .
$$

We see that Eq. (39) is an efficient bound on the integrity of $G_{3}^{1}$ if the given $\alpha_{p, q}^{d}$ 's are small enough, that is when it can be guaranteed that the variables are not too positively correlated. For small values or the maximum risk parameters, one proves that Eqs. (36) or (39) are generally the bests bounds that can be found on the integrity of the predicate $G_{n}^{f}$ when no other assumption is made on the stochastic properties of the variables. In the case of three variables for example, it can be shown that Eq. (39) is the largest lower bound on the probability of $G_{3}^{1}\left(x_{1}, x_{2}, x_{3}\right)$ when $\alpha_{1,2}^{d}+\alpha_{2,3}^{d}+\alpha_{3,1}^{d} \leq 1$ and $\alpha_{p, q}^{d}+\alpha_{r, p}^{d} \leq \alpha_{p}$ for all circular permutations $(p, q, r)$ of $\{1,2,3\}$.

The next section does the transition from the propositional logic to interval calculus. It shows how the integrity bounds found for Boolean functions also apply to interval functions when confidence degrees are assigned to the intervals.

\section{Confidence intervals}

This section tackles the problem of estimation of an unknown real parameter $\theta$ from the observations provided by a series of sensors. The observations consist of confidence intervals, placed around the actual scalar measurements of $\theta$ collected by the sensors. In this study, an interval is said to be valid if it contains the parameter $\theta$, while a set of intervals are compatible if their intersection is non-empty.

\subsection{From Boolean variables to interval integrities}

We consider $n$ ordered intervals $i_{1}, \ldots, i_{n}$, in which the parameter $\theta$ is certified to lie with certain probabilities. The set of the $n$ collected intervals is denoted by $I_{n}=\left\{i_{1}, \ldots, i_{n}\right\}$. For $k=1, \ldots, n$, we call $a_{k}$ and $b_{k}$ respectively the lower and upper limits of interval $i_{k}$ :

$$
i_{k}=\left[a_{k}, b_{k}\right] .
$$

To each interval $i_{k}(k=1, \ldots, n)$ we associate a Boolean variable $x_{k}$ expressing the validity of the interval :

$$
x_{k} \equiv \theta \in i_{k} .
$$

Variable $x_{k}$ takes the value true iif the corresponding interval $i_{k}$ contains $\theta$. The mode $\phi \in \Phi$ represents the uncertainties on the statistical performance of the sensors. We suppose that the obvious sensor failures are rejected beforehand through a fault detection process, which ensures that the remaining modes $\phi$ belong to a subset $\Phi_{0} \subset \Phi$. It is assumed that safe lower bounds on the probabilities that the parameter $\theta$ lies in the intervals are known inside $\Phi_{0}$, so that the probabilities of the Boolean variables $x_{k}$ are themselves bounded. These bounds are specified by equations of the form (4), with $x=x_{k}(k=1, \ldots, n)$. Eq. (41) involves the following correspondences between the set operators $(\cap, \cup)$ on one hand, and the propositional logic operators $(\wedge, \vee)$ on the other :

$$
\begin{aligned}
& x_{1} \wedge x_{2} \equiv \theta \in\left(i_{1} \cap i_{2}\right), \\
& x_{1} \vee x_{2} \equiv \theta \in\left(i_{1} \cup i_{2}\right) .
\end{aligned}
$$

The validity of the intersection of two intervals is equivalent to the conjunction of the corresponding Boolean variables, and the validity of their (set) union to the disjunction of these variables. As a consequence, the integrity bounds $(7,8)$ issued from the Boole-Fréchet bounds are the worst-case bounds on the probability to find $\theta$ in the union and intersection of two intervals respectively, when no information is known on the correlations of the interval faults. Otherwise, when the interval faults are correlated, these correlations can be expressed through the couples $\left(\alpha^{c}, \alpha^{d}\right)$ using the bounds of Eqs. $(13,14)$.

In addition, we mention the interval union $i_{1} \sqcup i_{2}$ of two intervals $i_{1}=\left[a_{1}, b_{1}\right]$ and $i_{2}=\left[a_{2}, b_{2}\right]$, which is a specific operator of interval arithmetic, and defined as the interval hull of these intervals :

$$
i_{1} \sqcup i_{2}=\left[\min \left(a_{1}, a_{2}\right), \max \left(b_{1}, b_{2}\right)\right] .
$$

The interval union has no direct equivalent in the Boolean setting. Though the interval union of two intervals always contains their set union, hence any integrity bounds for the set union of intervals can be considered as correct integrity bounds for the interval union as well.

\subsection{Fault-tolerant interval functions}

This section presents various fault-tolerant interval functions and uses the results of Sec. 3.3 to derive 
bounds on the probability that they contain the parameter $\theta$. We define $\mathcal{G}_{n}^{f}\left(I^{n}\right)$ as the set of points that belong to at least $n-f$ of the $n$ intervals $i_{1}, \ldots, i_{n}$ :

$$
\mathcal{G}_{n}^{f}\left(I^{n}\right)=\underset{\left\{k_{1}, \ldots, k_{n-f}\right\} \in \mathcal{C}_{n-f}^{k}}{\bigcup} i_{k_{1}} \cap \ldots \cap i_{k_{n-f}},
$$

with $I^{n}=\left\{i_{1}, \ldots, i_{n}\right\}$ and $0 \leq f \leq n-1$. It is easy to see that $\mathcal{G}_{n}^{f}\left(I^{n}\right)$ is also the set of points that verify the predicate function $G_{n}^{f}$ when the variables $x_{k}$ receive the Boolean values of the statements $\theta \in i_{k}(k=1, \ldots n)$. As a consequence, the set $\mathcal{G}_{n}^{f}\left(I^{n}\right)$ only gets larger with increased values of $f$. Indeed, Eq. (17) becomes

$$
\mathcal{G}_{n}^{f}\left(I^{n}\right) \subset \mathcal{G}_{n}^{f+1}\left(I^{n}\right) .
$$

Using the alternate definition (24) of $G_{n}^{f}\left(X^{n}\right)$, we also find, for $0 \leq f \leq n-1$,

$$
\mathcal{G}_{n}^{f}\left(I^{n}\right)=\bigcap_{\left\{k_{1}, \ldots, k_{f+1}\right\} \in \mathcal{C}_{n}^{f+1}} i_{k_{1}} \cup \ldots \cup i_{k_{f+1}} .
$$

In practice, the set of points provided by $\mathcal{G}_{n}^{f}$ is identical to the function proposed in [4]. Though there exists a conceptual difference as the function $\mathcal{G}_{n}^{f}$ does not take into account the posterior aspect of the intervals and makes no difference in its definition between empty and non-empty a posteriori observed intersections.

A lower bound on the worst-case probability that the unknown parameter $\theta$ lies in the set of points $\mathcal{G}_{n}^{f}\left(I^{n}\right)$ is given by Eq. (35), where variables $x_{k}$ are defined by (41). When the interval faults are independent, Eq. (35) reduces to

$$
P\left(\mathcal{G}_{n}^{f}\left(I^{n}\right)\right) \in\left[1-\sum_{\left\{k_{1}, \ldots, k_{f+1}\right\} \in \mathcal{C}_{n}^{f+1}} \alpha_{k_{1}} \ldots \alpha_{k_{f+1}}, 1\right],
$$

where $\alpha_{k}$ is the maximum risk of interval $i_{k}$ $(k=1 \ldots, n)$. Again, the maximum risk associated with $\mathcal{G}_{n}^{f}\left(I^{n}\right)$ is of order $f+1$ in $\alpha_{k}$ 's. It is possible to show that the bounds of Eq. (48) are the best possible bounds for small values of the risks $\alpha_{k}$. In the example of Fig. 1, four intervals $i_{1}, i_{2}, i_{3}, i_{4}$ are observed, and function $\mathcal{G}_{4}^{f}\left(I^{4}\right)$ is depicted for $f=1,2,3$. In this case, the four intervals are incompatible (their intersection is empty) and we have $\mathcal{G}_{4}^{0}\left(I^{4}\right)=\emptyset$. It can be seen that when the intervals are not compatible, function $\mathcal{G}_{n}^{f}$ may lead to a non-connex set of points. In the configuration of Fig. 1 for example, $\mathcal{G}_{4}^{2}\left(I^{4}\right)$ is made of two disjoint intervals. This property may represent a drawback in everyday applications, where it is generally more convenient to handle the pair of limits of a single interval than unions of intervals. Interval-valued functions, such as the function proposed by Marzullo in [1], are therefore more popular in the literature.

Marzullo's interval-valued function $\mathcal{M}_{n}^{f}$ is defined as the largest interval whose edges lie in the intersection of at least $n-f$ different intervals. The function $\mathcal{M}_{n}^{f}$ is in fact the intervall hull of $\mathcal{G}_{n}^{f}$, that is the smallest interval that contains $\mathcal{G}_{n}^{f}$. Following the syntax conventions of [17], we write

$$
\mathcal{M}_{n}^{f}\left(I^{n}\right)=\left[\mathcal{G}_{n}^{f}\left(I^{n}\right)\right]=\bigsqcup_{\left\{k_{1}, \ldots, k_{n-f}\right\} \in \mathcal{C}_{n-f}^{k}} i_{k_{1}} \cap \ldots \cap i_{k_{n-f}},
$$

Figure 1: Representation of $\mathcal{G}_{4}^{k}, \mathcal{M}_{4}^{k}$ and $\mathcal{F}_{4}^{k}$ with incompatible intervals, $n=4, k=1,2,3$.

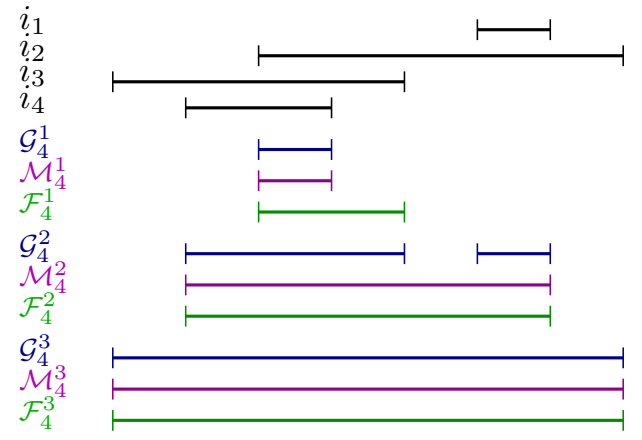

$0 \leq f \leq n-1$. From Eq. (47), we find

$$
\mathcal{M}_{n}^{f}\left(I^{n}\right)=\bigcap_{\left\{k_{1}, \ldots, k_{f+1}\right\} \in \mathcal{C}_{n}^{f+1}} i_{k_{1}} \sqcup \ldots \sqcup i_{k_{f+1}} .
$$

The most important feature of functions $\mathcal{G}_{n}^{f}$ and $\mathcal{M}_{n}^{f}$ is tolerance with respect to $f$ faulty input intervals. We refer the reader interested by detailed validity and precision properties of $\mathcal{M}_{n}^{f}$ to [1] and [18].

Suppose now the observed intervals are compatible, this happens when the lower limits of the intervals are all inferior to the upper limits. If we consider the alternate definition of function $\mathcal{M}_{n}^{f}$ given in Eq. (50), it is easy to see that $\mathcal{M}_{n}^{f}$ is in fact the interval limited by the $(f+1)$-th largest lower interval limit, denoted by $(f+1)-\max \left\{a_{1}, \ldots, a_{n}\right\}$, and the $(f+1)$-th smallest upper limit, denoted by $(f+1)-\min \left\{b_{1}, \ldots, b_{n}\right\}$. The latter observation leads to considering the faulttolerant function $\mathcal{F}_{n}^{f}$ introduced by U. Schmid in [6] :

$\mathcal{F}_{n}^{f}\left(I^{n}\right)=\left[(f+1)-\max \left\{a_{1}, \ldots, a_{n}\right\},(f+1)-\min \left\{b_{1}, \ldots, b_{n}\right\}\right]$.

Unlike the Marzullo function, $\mathcal{F}_{n}^{f}$ has the property to respect a Lipschitz condition for the interval lengths, which proves to be a very useful result in clock synchronization problems as pointed out in [2]. It is known that the Marzullo function $\mathcal{M}_{n}^{f}$ is always included in the Schmid function $\mathcal{F}_{n}^{f}$. Indeed, since the edges of $\mathcal{M}_{n}^{f}\left(I^{n}\right)$ belong to at least $n-f$ intervals, they must lie to the right of at least $n-f$ lower interval limits and to the left of at least $n-f$ lower limits, hence between the edges $(f+1)-\max \left\{a_{1}, \ldots, a_{n}\right\}$ and $(f+1)-\min \left\{b_{1}, \ldots, b_{n}\right\}$ of $\mathcal{F}_{n}^{f}\left(I^{n}\right)$. Moreover, by definition of the Marzullo function, the set of points $\mathcal{G}_{n}^{f}\left(I^{n}\right)$ is always included in the corresponding Marzullo interval $\mathcal{M}_{n}^{f}\left(I^{n}\right)$, the two functions providing exactly the same set of points when $\mathcal{G}_{n}^{f}\left(I^{n}\right)$ is connex. In summary, we have the following inclusions :

$$
\mathcal{G}_{n}^{f}\left(I^{n}\right) \subset \mathcal{M}_{n}^{f}\left(I^{n}\right) \subset \mathcal{F}_{n}^{f}\left(I^{n}\right),
$$

which give, in terms of probabilities,

$$
P_{\phi}\left(\theta \in \mathcal{G}_{n}^{f}\left(I^{n}\right)\right) \leq P_{\phi}\left(\theta \in \mathcal{M}_{n}^{f}\left(I^{n}\right)\right) \leq P_{\phi}\left(\theta \in \mathcal{F}_{n}^{f}\left(I^{n}\right)\right),
$$

for all $\phi \in \Phi_{0}$. The inclusion hierarchy of functions $\mathcal{G}_{n}^{f}$, $\mathcal{M}_{n}^{f}$ and $\mathcal{F}_{n}^{f}$ can be verified on Fig. 1 . The consequence of Eq. (53) is that the probability bounds of Eq. (48) for 
the function $\mathcal{G}$ are also valid for $\mathcal{M}$ and $\mathcal{F}$. Moreover, in a worst-case setting, the bounds of Eq. (48) are the best-possible bounds if one can not provide additional statistics on the mutual orientations of the interval faults. Indeed, under the worst-case mode $\hat{\phi}$, the faulty intervals are always situated on the same side of the parameter $\theta$, which is contained either by the three functions $\mathcal{G}_{n}^{f}, \mathcal{M}_{n}^{f}, \mathcal{F}_{n}^{f}$ or by none.

Proof. It is easy to admit that the validity of $\mathcal{G}_{n}^{f}$ depends only on the number of interval faults, not on their respective orientations. Hence, the worst-case mode for $\mathcal{M}$ and $\mathcal{F}$ is the mode that minimizes the validity probabilities of $\mathcal{M}_{n}^{f}$ and $\mathcal{F}_{n}^{f}$, given a lower bound on the validity probability of $\mathcal{G}_{n}^{f}$. Suppose that under mode $\hat{\phi}$ the interval faults always occur on the same side of $\theta$, say on the left side. Then all the lower limits $a_{k}$ are always inferior or equal to $\theta$. If $\mathcal{G}_{n}^{f}$ does not contain $\theta$, then $\theta$ lies to the right of $\mathcal{G}_{n}^{f}$ and $\left[\mathcal{G}_{n}^{f}\right]$, thus $\mathcal{M}_{n}^{f}$ does not contain $\theta$ either. This also implies that the number of interval faults exceeds $f$, leaving at least $f+1$ upper interval limits to the left of $\theta$, including the upper edge of $\mathcal{F}_{n}^{f}$. In summary, we have $P_{\hat{\phi}}\left(\theta \in \mathcal{G}_{n}^{f}\left(I^{n}\right)\right)=P_{\hat{\phi}}\left(\theta \in \mathcal{M}_{n}^{f}\left(I^{n}\right)\right)=P_{\hat{\phi}}\left(\theta \in \mathcal{F}_{n}^{f}\left(I^{n}\right)\right)$. The fault orientation issue is illustrated and discussed in Sec. 5 in a numerical example.

\section{Tests}

This section illustrates the performances of the faulttolerant functions $\mathcal{G}, \mathcal{M}$ and $\mathcal{F}$. The functions are tested with three independent sensors $(n=3)$. The sensors provide the confidence intervals $i_{1}, i_{2}$, and $i_{3}$ centered on the measurements of a same parameter $\theta$. We assume that the following integrity constraints are given for these intervals

$$
P_{\phi}\left(\theta \in i_{k}\right) \in[1-\alpha, 1],
$$

for $k=1,2,3$. Since the sensors are independent, it is assumed that

$$
P_{\phi}\left(\theta \in\left(i_{k} \cup i_{l}\right)\right) \in\left[1-\alpha^{2}, 1\right],
$$

where $(k, l)=(1,2),(2,3),(3,1)$. The set $\Phi_{0}$ is defined as the set of all the modes that verify the two integrity constraints above.

Now, we will analyse a particular mode $\phi_{1}$ of $\Phi_{0}$. The mode $\phi_{1}$ is chosen such that the midpoints of the intervals are independent variables with same Gaussian density function $f_{\phi_{1}}$ centered around $\theta$ with standard deviation $\sigma=1$. The length of the confidence intervals is chosen constant, equal for the three sensors, and such that

$$
P_{\phi_{1}}\left(\theta \in i_{k}\right)=1-\alpha,
$$

for $k=1,2,3$. For $(k, l)=(1,2),(2,3),(3,1)$, we have,

$$
P_{\phi_{1}}\left(\theta \in\left(i_{k} \cup i_{l}\right)\right)=1-\alpha^{2} \text {. }
$$

The statistics on the fault frequencies for $10^{7}$ simulations are depicted in Table 1 for $\alpha=0.1$ and $f=1$, and compared to the upper bound $3 \alpha^{2}$ given by Eq. (48). It can be seen that, under $\phi_{1}$, the worst-case risk is
Table 1: Fault rates and average lengths, noncorrelated fault orientations, $n=3, f=1, \alpha=0.1$.

\begin{tabular}{|c|c|c|c|}
\hline & w.c. risk & fault rate & mean length \\
\hline $\mathcal{G}_{3}^{1}$ & $3 \times 10^{-2}$ & $2.80 \times 10^{-2}$ & 3.2665 \\
\hline $\mathcal{M}_{3}^{1}$ & $3 \times 10^{-2}$ & $1.68 \times 10^{-2}$ & 3.2855 \\
\hline $\mathcal{F}_{3}^{1}$ & $3 \times 10^{-2}$ & $1.45 \times 10^{-2}$ & 3.2897 \\
\hline
\end{tabular}

Table 2: Fault rates and average lengths, perfectlycorrelated fault orientations, $n=3, f=1, \alpha=0.1$.

\begin{tabular}{|c|c|c|c|}
\hline & w.c. risk & fault rate & mean length \\
\hline $\mathcal{G}_{3}^{1}$ & $3 \times 10^{-2}$ & $2.80 \times 10^{-2}$ & 3.28950 \\
\hline $\mathcal{M}_{3}^{1}$ & $3 \times 10^{-2}$ & $2.80 \times 10^{-2}$ & 3.28966 \\
\hline $\mathcal{F}_{3}^{1}$ & $3 \times 10^{-2}$ & $2.80 \times 10^{-2}$ & 3.28971 \\
\hline
\end{tabular}

close to the actual risk for function $\mathcal{G}\left(3 \alpha^{2}-2 \alpha^{3}\right)$, but is very pessimistic for the Marzullo and Schmid $\left(3 / 2 \alpha^{2}-1 / 2 \alpha^{3}\right)$ functions. The last column of the table shows the gradation of the mean lengths of the three functions, where we considered that the length of the non-connex function $\mathcal{G}$ was equal to the sum of the lengths of its components. We imagine now another mode $\phi_{2}$ similar to $\phi_{1}$ with the difference that $\phi_{2}$ retains only positive values for the measurement errors. Under $\phi_{2}$, the midpoints of the intervals are independent variables following the same probability distribution of density

$$
f_{\phi_{2}}(t)=\mid \begin{array}{ll}
0 & \text { if } t<\theta \\
2 f_{\phi_{1}}(t) & \text { if } t \geq \theta
\end{array} .
$$

Since $\phi_{2}$ verifies Eqs. $(56,57), \phi_{2}$ belongs to $\Phi_{0}$. This time, however, the fault orientations are perfectly correlated. The consequence of this can be observed in Table 2 : the fault rates of the three functions $\left(3 \alpha^{2}-2 \alpha^{3}\right)$ are equal, and close to the worst-case risk. This confirms the optimality of Eq. (48) in a worst-case setting. More elaborate tests covering the issue of correlated sensors and the integrity/precision trade-off will be reported in future publications.

\section{Discussion}

The integrity certification possibilities presented in this study are particularly relevant for all applications demanding high safety requirements. These applications include, inter alia, mobile positioning, railway signaling systems, automobile indicator lights, emergency shut-down systems, turbine control, or medical devices. Besides the risk assessment of interval functions, the generic character of the Boolean approach suggests other potential application fields where the inputs are not necessarily unidimensional confidence intervals on the estimated parameter. In triangularization problems for instance, the sensors do not provide confidence intervals on the mobile's position but on the distances to reference points. In particular, in the problem of GPS pseudo-range estimation from a constellation of $n$ 
satellites, the function $G_{n}^{f}$ leads directly to safe confidence intervals for the position (or speed) of the mobile for sufficient difference $n-f$. The approach presented in this study offers more flexibility than recent safe satellite positioning methods, which require the prior derivation of independent intervals on the position (or speed) by combination of subsets of satellites before the interval union or any interval function can be applied to these intervals [3].

\section{Conclusion}

We presented in this study a novel approach to the risk assessment of robust sensor fusion methods based on fault-tolerant interval functions, in the context of applications requiring high levels of integrity. Adopting a Boolean framework, we symbolized the uncertain validity of each confidence interval by a Boolean variable. We showed how to express constraints on the confidence degrees of the intervals and on the dependencies between interval faults in terms of probability bounds on these Boolean variables. We explained how the Boole-Fréchet bounds and the theory of probabilistic inference allowed to derive safe integrity bounds on given combinations of intervals. In particular, we proposed a predicate formula $G_{n}^{f}$ offering an interesting trade-off between the properties of the conjunction (gain in integrity) and disjunction (gain in accuracy) operators. The predicate $G_{n}^{f}$ proved to be a non-connex version of Marzullo's fault-tolerant interval function extended to propositional logic. Moreover, the formula $G_{n}^{f}$ allowed to derive safe integrity bounds for the fault-tolerant interval functions proposed by Marzullo and Schmid. The relative performances of the functions, and the relevance of the integrity bounds provided were illustrated in a numerical example.

Acknowledgements. Thanks to Multitel ASBL, the Data Fusion Group at Multitel for their valuable suggestions (regarding Sec. 6 in particular), Andreea, Raphaël.

\section{References}

[1] K. Marzullo. Maintaining the time in a distributed system. An example of a loosely-coupled distributed service. $\mathrm{PhD}$ thesis, Dept. of Electrical Engineering, Stanford University, Stanford, Ca., 1984.

[2] L. Lamport. Synchronizing time servers. Technical Report 18, Digital Equipment Corporation Systems Research Center, Palo Alto, CA (USA), 1987.

[3] I. Nikiforov and F. Choquette. Integrity monitoring for safe train positioning using GNSS. In 11th IAIN World Congress, pages 1-20, Berlin, Germany, October 2003.

[4] Y. Zhu and B. Li. Optimal interval estimation fusion based on sensor interval estimates and con- fidence degrees. In Multisensor, Multisource Information Fusion: Architectures, Algorithms, and Applications 2003. Proceedings of the SPIE, volume 5099, pages 268-278, april 2003.

[5] E.T. Jaynes. Probability theory : the logic of science. Cambridge University Press, 2003.

[6] U. Schmid and K. Schossmaier. How to reconcile fault-tolerant interval intersection with the Lipschitz condition. Distributed Computing, 14(2):101-111, 2001.

[7] S. Ferson, V. Kreinovich, L. Ginzburg, D.S. Myers, and K. Sentz. Constructing probability boxes and Dempster-Shafer structures, technical report SAND2002-4015. Sandia National Laboratory, 2003.

[8] Arnold Neumaier. Clouds, fuzzy sets and probability intervals. Reliable computing, 10:249-272, 2004.

[9] M. Fréchet. Généralisation du théorème des probabilités totales. Fundamenta Mathematica, 25:379-387, 1935.

[10] S. Ferson, J. Hajagos, D. Berleant, J. Zhang, W. T. Tucker, L. Ginzburg, and W. Oberkampf. Dependence in Dempster-Shafer theory and probability bounds analysis, technical report SAND2004-3072. Sandia National Laboratory, 2004.

[11] B.P. Wise and M. Henrion. A framework for comparing uncertain inference systems to probability. In L. N. Kanal and J. F. Lemmer, editors, Uncertainty in Artificial Intelligence, pages 69-83, Amsterdam, The Netherlands, 1986. North-Holland.

[12] R.E. Moore. Interval Analysis. Prentice-Hall, Inc., Englewood Cliffs, NJ, 1966.

[13] R.C. Williamson. Probabilistic Arithmetic. PhD thesis, Dept. of Electrical Engineering, University of Queensland, Brisbane, Australia, 1989.

[14] R.E. Moore. Methods and Applications of Interval Analysis, volume 2. SIAM Studies in Applied Mathematics, Philadelphia, PA, 1979.

[15] T. Hailperin. Probability logic. Notre Dame Journal of Formal Logic, 25(3):198-212, 1984.

[16] M. Jaeger. Automatic derivation of probabilistic inference rules. Int. J. Approx. Reasoning, 28(1):1-22, 2001.

[17] L. Jaulin, M. Kieffer, O. Didrit and E. Walter. Applied Interval Analysis, with Examples in Parameter and State Estimation, Robust Control and Robotics. Springer-Verlag, London, 2001.

[18] U. Schmid. Orthogonal accuracy clock synchronization. Chicago Journal of Theoretical Computer Science, 2000. 